\title{
A NOTE ON GENERALIZED CAUCHY-SCHWARZ INEQUALITY
}

\section{SONGTING YIN}

Abstract. We generalize the well-known Cauchy-Schwarz inequality involving any number of real or complex matrices, and also give a necessary and sufficient condition for the equality. This is an improvement of the two recent literatures due to N. Harvey and D. Choi.

Mathematics subject classification (2010): 26D15.

Keywords and phrases: Cauchy-Schwarz inequality, real matrix, complex matrix.

\section{REFERENCES}

[1] N. HARVEY, A generalization of the Cauchy-Schwarz inequality involving four vectors, J. Math. Inequal., 2015, 9 (2): 489-491.

[2] D. CHOI, A generalization of the Cauchy-Schwarz inequality, J. Math. Inequal., 2016, 10 (4): 10091012. 